\title{
Microscopic Origin and Universality Classes of the Efimov Three-Body Parameter
}

\author{
Pascal Naidon ${ }^{1}$, Shimpei Endo ${ }^{2}$, and Masahito Ueda ${ }^{2}$ \\ ${ }^{1}$ RIKEN Nishina Centre, RIKEN, Wakō 351-0198, Japan, and \\ ${ }^{2}$ Department of Physics, University of Tokyo, 7-3-1 Hongō, Bunkyō-ku, Tōkyō 113-0033, Japan
}

(Dated: October 30, 2018)

\begin{abstract}
The low-energy spectrum of three particles interacting via nearly resonant two-body interactions in the Efimov regime is set by the so-called three-body parameter. We show that the three-body parameter is essentially determined by the zero-energy two-body correlation. As a result, we identify two classes of two-body interactions for which the three-body parameter has a universal value in units of their effective range. One class involves the universality of the three-body parameter recently found in ultracold atom systems. The other is relevant to short-range interactions that can be found in nuclear physics and solid-state physics.
\end{abstract}

The Efimov effect is a universal low-energy quantum phenomenon, which was originally predicted in nuclear physics [1] and has rekindled considerable interest since its experimental confirmation with ultracold atoms 22 22. It is also expected to occur in solid-state physics 23, 24. This universality stems from the effective three-body attraction that occurs between particles interacting with nearly resonant short-range interactions. As a result of this attraction, three particles may bind even when the interaction is not strong enough to bind two particles. Furthermore, an infinite series of such three-body bound states exists near the unitary point where the interaction is resonant, i.e. where a two-body bound state appears and the $s$-wave scattering $a$ length diverges. The typical three-body energy spectrum for such systems is represented in Fig. 11 in units of inverse length. Near zero energy and large scattering lengths, the three-body spectrum is invariant under a discrete scaling transformation by a universal factor $e^{\pi / s_{0}} \approx 22.7$ for identical bosons, where $s_{0} \approx 1.00624$ characterises the strength of the three-body attraction.

A notable consequence of the Efimov effect is the existence of another physical scale beyond the two-body scattering length to fix the low-energy properties of the system. This scale is known as the three-body parameter. In zero-range models, it manifests itself as the necessity to introduce a momentum cutoff or a three-body boundary condition. It can be characterised, for instance, by the scattering length $a_{-}$at which a trimer appears or by its binding wave number $\kappa$ at unitarity, as indicated in Fig. 1. Because of the discrete scaling invariance, it is defined up to a power of $e^{\pi / s_{0}}$. In this Letter, we will focus on the ground Efimov state, which slightly deviates from the discrete-scaling-invariant structure, but is more easily observed and computed, and still reveals the essence of the physics behind the three-body parameter.

Three important questions can be raised concerning the three-body parameter. Is there a simple mechanism that determines the three-body parameter from the microscopic interactions? What is the microscopic length scale which determines the three-body parameter? Finally, if there is such a length scale, what are the conditions for the three-body parameter to be related to that length scale through a universal dimensionless constant,

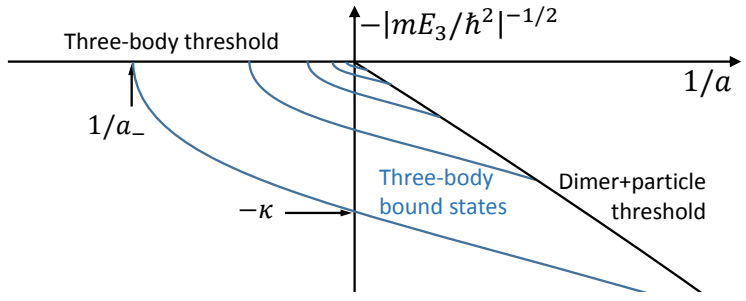

Figure 1: Schematic Efimov plot: three-body energy $E_{3}$ scaled as an inverse length as a function of the inverse scattering length $1 / a$. The arrows indicate the scattering length $a_{-}$at which an Efimov trimer state appears, and its binding wave number $\kappa$ at unitarity $(a \rightarrow \infty)$, either of which serves as a measure of the three-body parameter.

as suggested by experimental observations Gross et al. [15], Berninger et al. 17] and recent calculations Wang et al. 25]? This Letter answers these three questions for systems of identical bosons (or three distinguishable fermions with equal mass) where the resonant interaction can be described by a single scattering channel. In ultracold atoms experiments, the interaction is made resonant by using magnetic Feshbach resonances [26. The present results are thus applicable to the case of broad Feshbach resonances, which are dominated by their open channel, but not to narrow Feshbach resonances, which are strongly affected by their closed channel [27].

The question of the physical mechanism setting the three-body parameter was addressed in Refs. 25, 28] for van der Waals interactions, which decay as $1 / r^{6}$ at large interparticle distance $r$. The numerical investigation of Ref. 25] found that in the hyperspherical formalism, in addition to the three-body Efimov attraction at large distances, a three-body repulsion appears at short distances. The distance at which this repulsion appears is comparable to the size of the van der Waals tail of the potential, thus preventing the system from probing the details of the interaction at shorter distances. Therefore, the value of the three-body parameter is set by the van der Waals length associated with that tail. The authors of Ref. 25] remarked that this three-body repulsion is not explained by quantum reflection, as originally suggested in Ref. [29], but attributed it to an increase in 
kinetic energy due to the squeezing of the hyperangular wave function into a smaller volume caused by the suppression of two-body probability inside the well or the repulsive core of the two-body potential. This point was confirmed and clarified in Ref. [28] where the kinetic energy was shown to originate from an abrupt change of the geometry of the three-particle system caused by the twoparticle exclusion in the van der Waals region. At large separation, the system has indeed an elongated geometry due to its Efimov nature, but it must deform to an equilateral configuration to accommodate for the mutual exclusion between the particles. Reference [28] showed that this deformation causes a nonadiabatic increase in kinetic energy that manifests itself as a three-body repulsive barrier. This phenomenon could be reproduced by simple models involving only the knowledge of the pair correlation causing the mutual exclusion between two particles at short separations.

One may wonder whether these findings extend to other physical systems. Indeed, the same deformation mechanism is expected to occur in systems for which the two-body interactions tend to suppress the two-body probability at short distance. Thus, pair correlation should provide the essential information that determines the three-body parameter and energy of the three-body system.

To investigate the role of pair correlation, we use a simple model that reproduces the pair correlation and can be solved exactly for three particles, and then compare it with full exact calculations. We cannot use a zero-range model because such a model would reproduce the asymptotically free part of the two-body wave function (i.e. the on-shell $T$-matrix elements), but not its short-range correlation (i.e. the off-shell $T$-matrix elements). We thus follow the approach introduced in Ref. [28], where the interaction is modelled by a separable potential [30, $\hat{V}=\xi|\chi\rangle\langle\chi|$, which retains much of the mathematical simplicity of a contact potential, while enabling us to reproduce any pair correlation at zero energy. Indeed, for a given zero-energy two-body $s$-wave radial wavefunction $u_{0}(r)$ with the asymptotic limit $1-\frac{r}{a}$, where $a$ is the scattering length, one can construct a separable potential reproducing this wave function exactly by choosing the following form (in momentum representation):

$$
\begin{gathered}
\chi(q)=1-q \int_{0}^{\infty} d r\left(1-\frac{r}{a}-u_{0}(r)\right) \sin (q r) \\
\xi=4 \pi\left(\frac{1}{a}-\frac{2}{\pi} \int_{0}^{\infty} d q|\chi(q)|^{2}\right)^{-1}
\end{gathered}
$$

This simple prescription reproduces the low-energy two-body physics, in particular the two-body bound state around the unitary limit $a \rightarrow \infty$. We construct separable potentials that reproduce the pair correlation of the various two-body potentials considered in Refs. 25] and 31. The three-body problem for a separable interaction can

\begin{tabular}{lllllll}
\hline Potential & $g_{3}$ & $g_{3}^{\prime}$ & $a_{-}$ & $a_{-}^{\prime}$ & $E_{3}$ & $E_{3}^{\prime}$ \\
\hline \hline Yukawa & 1.35 & 1.38 & -5.73 & -6.55 & -0.172 & -0.134 \\
Exponential & 1.17 & 1.16 & -10.7 & -11.0 & -0.047 & -0.042 \\
Gaussian & 2.12 & 2.14 & -4.27 & -4.47 & -0.236 & -0.223 \\
Morse $\left(r_{0}=1\right)$ & 0.294 & 0.295 & -12.3 & -12.6 & -0.0325 & -0.0299 \\
Morse $\left(r_{0}=2\right)$ & 0.205 & 0.205 & -16.4 & -16.3 & -0.0174 & -0.0166 \\
Pöschl-Teller $(\alpha=1)$ & 0.797 & 0.802 & -6.02 & -6.23 & -0.135 & -0.123 \\
\hline
\end{tabular}

Table I: Three-body properties obtained for various potentials considered in Ref. 31], where $g_{3}$ denotes the factor required to multiply the potential so that the ground-state Efimov trimer appears at the three-body threshold, $a_{-}$denotes the scattering length for that factor, and $E_{3}$ denotes the energy of the ground-state Efimov trimer at unitarity $(a \rightarrow \infty)$. The symbols without a prime indicate that the values are taken from Ref. 31, and those with a prime show our results based on the pair correlation using the separable model given by Eqs. (1) and (2). The same units as in Ref. 31] are used.

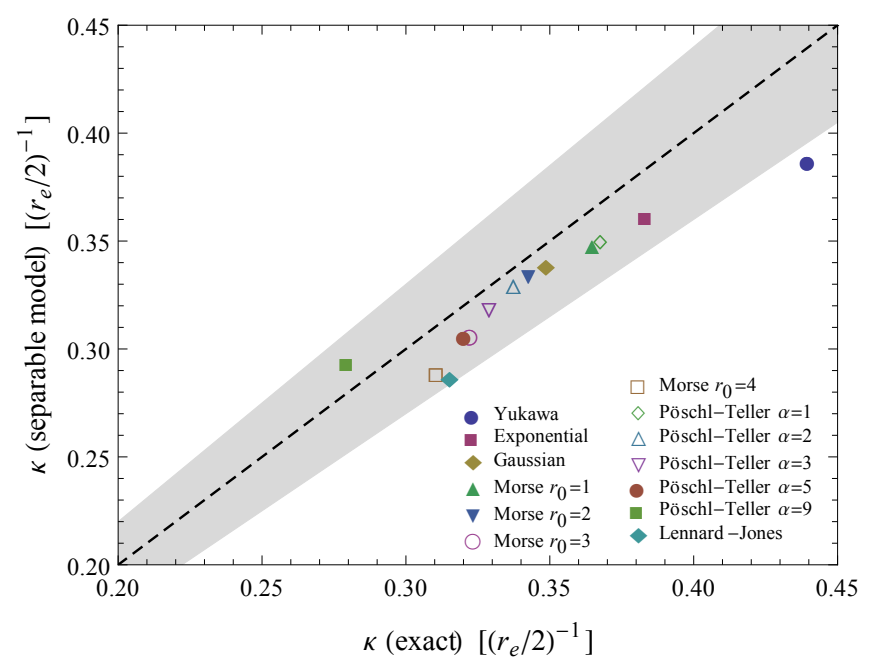

Figure 2: Binding wave number $\kappa$ of the ground-state trimer at unitarity (see Fig.1) calculated from the separable model in Eqs. (1) and (2) using the zero-energy pair wave function $\psi_{0}$, versus its exact value, for various two-body potentials. The exact values are taken from Ref. 25] for the Lennard-Jones potential (with only one bound state) and from Ref. 31] for all the other potentials. The binding wave number is expressed in units of the effective range $r_{e}$ of each potential, which is calculated exactly from Eq. 3. The shaded area represents the region of $10 \%$ or less deviation from the exact results.

be cast in the form of an integral equation in momentum space that can easily be solved numerically $28,32,33$. The results are shown in Table \ where we indicate the values $a_{-}$and $\kappa$ for the ground-state trimer. They agree with the exact calculations of Refs. 25] and 31 to within a few percents for each of these potentials. This can be checked in Fig. 2 where the value of $\kappa$ in our model is plotted against its exact value. The method presented here therefore appears as a simple and efficient way to estimate the three-body parameter, and more generally 
low-energy properties for various kinds of interaction potentials.

Now that we have established the connection between the pair correlation and the three-body parameter, we are in a position to ask which length scale in the pair correlation determines the three-body parameter. For most physical interactions, the major effect of pair correlations is to suppress probability at short distance with respect to the free wave. As discussed previously, this creates a three-body repulsion through the nonadiabatic deformation effect. Although the precise shape of the repulsive barrier depends on the particular two-body potential, it should be the length scale associated with the two-body suppression that sets the location of the three-body repulsion, and therefore the three-body parameter. This length scale is given by half the effective range $\frac{1}{2} r_{e}$ [42], which is the average size of the deviation between the asymptotic and fully correlated probability densities [34, 35]:

$$
\frac{1}{2} r_{e}=\int_{0}^{\infty} d r\left(\left(1-\frac{r}{a}\right)^{2}-u_{0}(r)^{2}\right)
$$

Thus for common interactions which tend to suppress the two-body probability within their range, $r_{e}$ is positive and the three-body parameter, expressed in the dimension of length, is on the order of $\frac{1}{2} r_{e}$. Note that the effective range is commonly featured as a term in the lowenergy expansion of the scattering phase shift (i.e. the on-shell $T$-matrix elements). However, we expect that it is not possible to find a connection between the threebody parameter and the effective range from a method which introduces the effective range in this manner, since this expansion concerns only on-shell scattering and does not describe the short-range correlation explicitly 43 .

We can now address the final question of whether there are classes of interactions for which the low-energy threebody physics is universally determined. It is clear that if the pair correlation is the same for a certain class of potentials, they must lead to the same three-body parameter. This is indeed the case for potentials with a powerlaw decaying tail $-C_{n} r^{-n}$, such as the van der Waals tail $-C_{6} r^{-6}$ relevant to the interaction between ground-state atoms. It is well known that the two-body wave functions in the tail of these potentials are universally described in terms of the length scale $r_{n}=\left(\frac{1}{n-2} \sqrt{m C_{n}} / \hbar\right)^{2 /(n-2)}$. If most of the probability amplitude is located in the tail region, which is the case if the short-range region is strongly repulsive or attractive, all these potentials lead to a similar zero-energy pair correlation that is known analytically:

$$
\begin{aligned}
& u_{0}(r)=\Gamma\left(\frac{n-1}{n-2}\right) \sqrt{x} J_{\frac{1}{n-2}}\left(2 x^{-\frac{n-2}{2}}\right) \\
& \quad-\frac{r_{n}}{a} \Gamma\left(\frac{n-3}{n-2}\right) \sqrt{x} J_{-\frac{1}{n-2}}\left(2 x^{-\frac{n-2}{2}}\right),
\end{aligned}
$$

where $\Gamma$ and $J_{\alpha}$ denote the gamma and Bessel functions, and $x=r / r_{n}$. The universality of the pair correlation

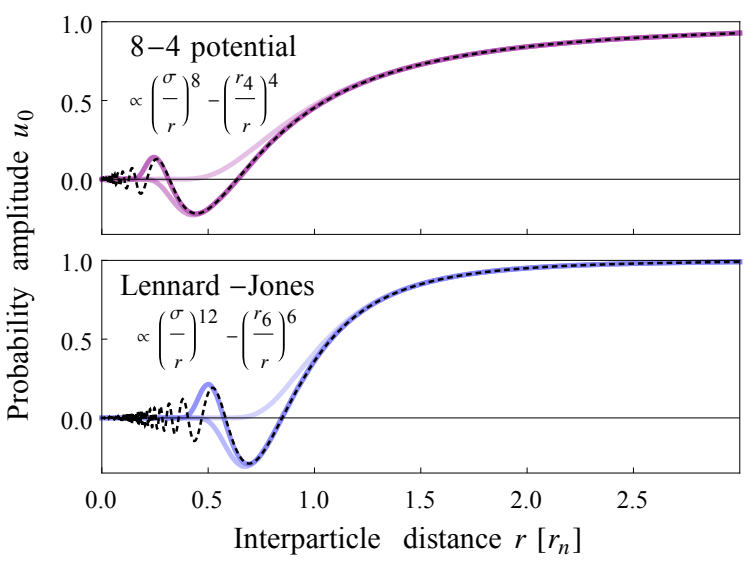

Figure 3: Pair correlation at unitarity for potentials decaying as power laws $-1 / r^{n}$. Top: the $8-4$ potential. Bottom: the Lennard-Jones potential. In each graph, the solid curves correspond in order of opacity to potential depths supporting respectively 1,2 , and $3 s$-wave bound states, which are obtained by adjusting the value of $\sigma$. The dashed curve represents the universal pair correlation in Eq. (4).

is illustrated in Fig. 3 for the $8-4$ and Lennard-Jones $(12-6)$ potentials of various depths. This gives a simple explanation of the observed universality of the threebody parameter in atomic systems ranging from light helium [22, 36] to heavy atoms under broad magnetic Feshbach resonances [15, 17]. Figure 5 shows the binding wave number $\kappa$ of the ground-state trimer for these power-law decaying potentials, evaluated using our separable potential method. For potential depths supporting more than one two-body bound state, the ground-state trimer is in fact a resonance in the particle-dimer continuum, but it manifests itself simply as a bound state in our model [44]. One can see that the value of $\kappa$ remains close to the one obtained from the universal pair correlation. In the particular case of a van der Waals tail, we obtain $a_{-}=-10.86(1) r_{6}$ and $\kappa=0.187(1) r_{6}^{-1}$ in good agreement with Ref. 25] and experimental observations [17, 37. Since the effective range is related to the van der Waals length $r_{6}$ through $r_{e}=\frac{4 \pi}{3 \Gamma(3 / 4)^{2}} r_{6} \approx 2.78947 r_{6}$, these results correspond to $a_{-}=-7.78(1) \times\left(\frac{1}{2} r_{e}\right)$ and $\kappa=0.261(1) \times\left(\frac{1}{2} r_{e}\right)^{-1}$.

There is a second class of potentials, which decay faster than any power law, such as the Yukawa potential and other typical nuclear potentials, as well as screened potentials found in solid-state physics. At first glance, the two-body wave functions for these potentials do not seem to exhibit any particular universality. However, if the potential features a deep attraction supporting many bound states, the effective range near unitarity is large. This means that when distances are expressed in units of $\frac{1}{2} r_{e}$, there is a sharp drop of probability in the two-body wave function near $r=1$, as represented in Fig. 4. It can be shown that this rescaled two-body wave function converges to a step function in the limit of strongly attractive 


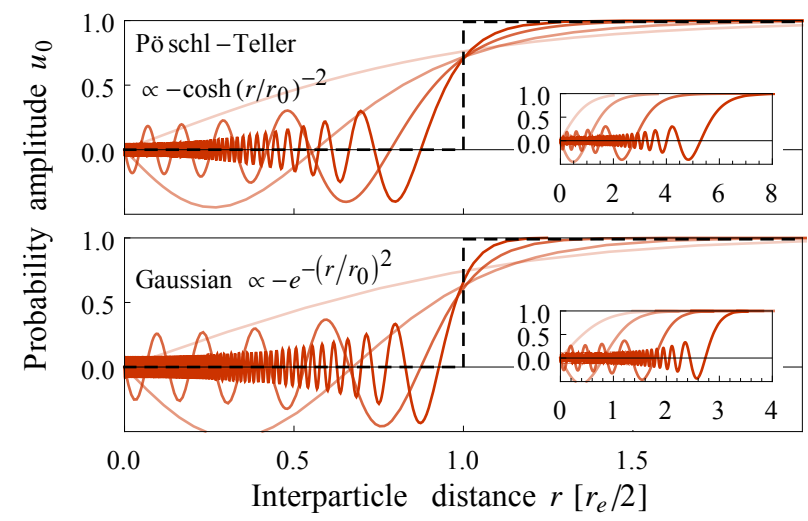

Figure 4: Pair correlation at unitarity for potentials decaying faster than any power law. Top: the Pöschl-Teller potential; bottom: the Gaussian potential. In each graph, the solid curves correspond in order of opacity to potential depths supporting respectively $1,2,10$, and $120 \mathrm{~s}$-wave bound states. The dashed lines show the universal pair correlation limit in Eq. 5). The distance is scaled in units of $\frac{1}{2} r_{e}$ in the main graphs, while it is shown in unscaled units of $r_{0}$ in the insets.

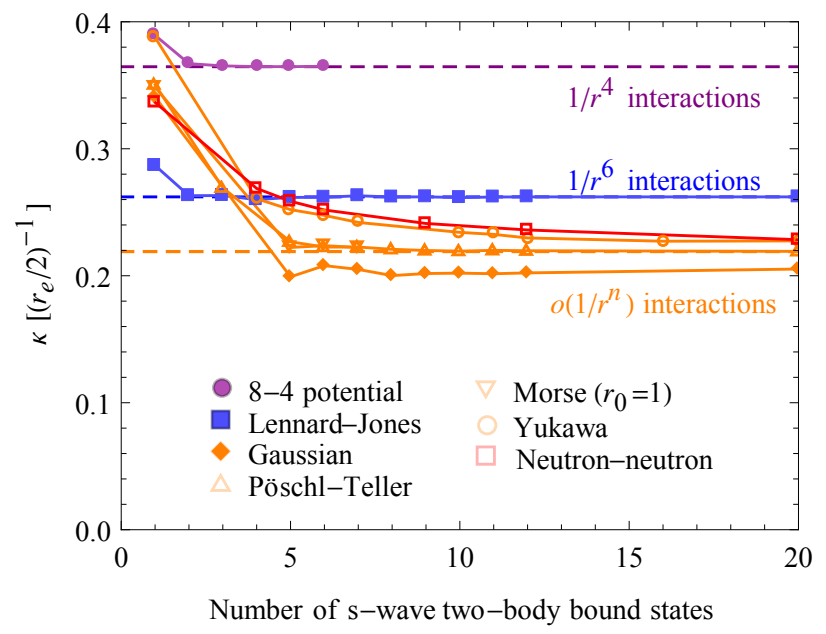

Figure 5: Binding wave number $\kappa$ of the ground-state Efimov trimer calculated from the separable model in Eqs. (1) and (2) for pair correlations corresponding to different two-body interactions, as a function of the depth of these potentials as measured by the number of $s$-wave two-body bound states. The dashed lines indicate from top to bottom the values obtained for the universal pair correlation in Eq. (4) with $n=4$ and $n=6$, and the universal pair correlation in Eq. (5), respectively. This figure shows how the three-body parameter converges differently and to different limits depending on the class of two-body interaction.

potentials [45]. In this sense, the three-body parameter is universally determined by the effective range of these potentials, and stems from the universal pair correlation limit:

$$
u_{0}(r)=\left\{\begin{array}{ll}
0 & \text { for } r<\frac{1}{2} r_{e} \\
1-\frac{r}{a} & \text { for } r \geq \frac{1}{2} r_{e}
\end{array} .\right.
$$

Figure 5 shows the trimer binding wave number $\kappa$ for some of these potentials, namely, the Gaussian potential, the Pöschl-Teller potential with $\alpha=1$, the Yukawa potential, the Morse potential with $r_{0}=1$ [31, as well as the neutron-neutron interaction potential in the ${ }^{1} S_{0}$ channel [38]. While none of these calculations correspond to a particular physical system, they capture the essence of the Efimov physics occurring in the symmetric channel of nuclear systems, such as the tritium nucleus. Each potential was scaled to reach unitarity, corresponding to different possible depths of the potential. One can see in Fig. 5 that as the depth of the potentials is increased, $\kappa$ converges to the value $\kappa=0.2190(1) \times\left(\frac{1}{2} r_{e}\right)^{-1}$ obtained for the two-body correlation in Eq. (5). The convergence is, however, very slow, because very deep potentials (supporting hundreds of bound states) are required for the pair correlation to approach Eq. (5).

Finally, one should note that there is a notable exception to these considerations. One might think that the square-well potential, which often lends itself to simple analytical treatments [39], is a useful model potential to investigate the physics of the three-body parameter. However, it turns out to be a special case which does not belong to the two classes discussed above. Even though it decays faster than any power law, it does not belong to the second class because of its absence of tail. In particular, the two-body wave function near unitarity shows no progressive drop of probability in the well, only steady oscillations which get faster as the depth of the well increases, and therefore does not converge to the function in Eq. (5). From this we conclude that this potential is not expected to reveal any universality of the three-body parameter.

To summarise, we have pointed out how the Efimov three-body parameter is deeply connected to the zeroenergy two-body correlation. This allows us to identify the two-body effective range as the relevant length scale setting the three-body parameter for the class of physical interactions which suppress two-body probability at short distance. However, it also shows that, unlike what was suggested in Ref. 25, this suppression of two-body probability does not lead to a single universal value of the three-body parameter in units of the effective range. Indeed we find two qualitatively distinct subclasses of interactions for which the value of the three-body parameter is universally determined. One corresponds to short-range two-body potentials decaying as a power law, relevant to atomic interactions, for which the three-body universality stems from the two-body universality. The other corresponds to very deep two-body potentials decaying faster than any power law, which lead to an abrupt two-body suppression. Typical interactions in nuclear physics decay faster than any power law but support only a few bound states, so that their three-body parameter 
does not reach this universal limit. In practice, however, one can expect the binding wave number $\kappa$ to be in the range $0.2 \sim 0.4 \times\left(\frac{1}{2} r_{e}\right)^{-1}$ for most physical interactions, and in particular close to $0.35 \times\left(\frac{1}{2} r_{e}\right)^{-1}$ for nuclear interactions supporting at most one bound state, as can be seen in Figs. 2 and 5. These conclusions are obtained for particles interacting through single-channel two-body interactions, and would not apply in the presence of significant three-body forces, or strongly energy- dependent resonant interactions such as narrow Feshbach resonances [27, 40, 41].

P. N. acknowledges support from RIKEN through the Incentive Research Project funding. S. E. acknowledges support from JSPS (Grant No. 237049). M. U. acknowledges support by Grants-in-Aid for Scientific Research (Kakenhi No. 22340114 and No. 22103005) and the Photon Frontier Network Program of MEXT of Japan.
[1] V. Efimov, Phys. Lett. B 33, 563 (1970).

[2] T. Kraemer, M. Mark, P. Waldburger, J. G. Danzl, C. Chin, B. Engeser, A. D. Lange, K. Pilch, A. Jaakkola, H.-C. Nägerl, et al., Nature (London) 440, 315 (2006).

[3] T. B. Ottenstein, T. Lompe, M. Kohnen, A. N. Wenz, and S. Jochim, Phys. Rev. Lett. 101, 203202 (2008).

[4] J. H. Huckans, J. R. Williams, E. L. Hazlett, R. W. Stites, and K. M. O'Hara, Phys. Rev. Lett. 102, 165302 (2009).

[5] J. R. Williams, E. L. Hazlett, J. H. Huckans, R. W. Stites, Y. Zhang, and K. M. O'Hara, Phys. Rev. Lett. 103, 130404 (2009).

[6] M. Zaccanti, B. Deissler, C. D'Errico, M. Fattori, M. Jona-Lasinio, S. Müller, G. Roati, and G. M. M. Inguscio, Nat. Phys. 5, 586 (2009).

[7] G. Barontini, C. Weber, F. Rabatti, J. Catani, G. Thalhammer, M. Inguscio, and F. Minardi, Phys. Rev. Lett. 103, 043201 (2009).

[8] A. N. Wenz, T. Lompe, T. B. Ottenstein, F. Serwane, G. Zürn, and S. Jochim, Phys. Rev. A 80, 040702 (2009).

[9] S. E. Pollack, D. Dries, and R. G. Hulet, Science 326, 1683 (2009).

[10] N. Gross, Z. Shotan, S. Kokkelmans, and L. Khaykovich, Phys. Rev. Lett. 103, 163202 (2009).

[11] S. Knoop, F. Ferlaino, M. Mark, M. Berninger, H. Schoebel, H.-C. Naegerl, and R. Grimm, Nat. Phys. 5, 227 (2009).

[12] T. Lompe, T. B. Ottenstein, F. Serwane, K. Viering, A. N. Wenz, G. Zürn, and S. Jochim, Phys. Rev. Lett. 105, 103201 (2010).

[13] T. Lompe, T. B. Ottenstein, F. Serwane, A. N. Wenz, G. Zürn, and S. Jochim, Science 330, 940 (2010).

[14] S. Nakajima, M. Horikoshi, T. Mukaiyama, P. Naidon, and M. Ueda, Phys. Rev. Lett. 105, 023201 (2010).

[15] N. Gross, Z. Shotan, S. Kokkelmans, and L. Khaykovich, Phys. Rev. Lett. 105, 103203 (2010).

[16] S. Nakajima, M. Horikoshi, T. Mukaiyama, P. Naidon, and M. Ueda, Phys. Rev. Lett. 106, 143201 (2011).

[17] M. Berninger, A. Zenesini, B. Huang, W. Harm, H.-C. Nägerl, F. Ferlaino, R. Grimm, P. S. Julienne, and J. M. Hutson, Phys. Rev. Lett. 107, 120401 (2011).

[18] F. Ferlaino, S. Knoop, M. Berninger, W. Harm, J. P. D'Incao, H.-C. Nägerl, and R. Grimm, Phys. Rev. Lett. 102, 140401 (2009).

[19] F. Ferlaino and R. Grimm, Physics 3, 9 (2010).

[20] O. Machtey, Z. Shotan, N. Gross, and L. Khaykovich, Phys. Rev. Lett 108, 210406 (2012).

[21] A. Zenesini, B. Huang, M. Berninger, S. Besler, H.-C. Nägerl, F. Ferlaino, R. Grimm, C. H. Greene, and J. von Stecher, New J. Phys. 15, 043040 (2013).

[22] S. Knoop, J. S. Borbely, W. Vassen, and S. J. J. M. F.
Kokkelmans, Phys. Rev. A 86, 062705 (2012).

[23] Y. Nishida, Y. Kato, and C. D. Batista, Nat. Phys. 9, 93 (2013).

[24] J. Omachi, T. Suzuki, K. Kato, N. Naka, K. Yoshioka, and M. Kuwata-Gonokami, Phys. Rev. Lett. 111, 026402 (2013).

[25] J. Wang, J. P. D'Incao, B. D. Esry, and C. H. Greene, Phys. Rev. Lett. 108, 263001 (2012).

[26] C. Chin, R. Grimm, P. S. Julienne, and E. Tiesinga, Rev. Mod. Phys. 82, 1225 (2010).

[27] D. S. Petrov, Phys. Rev. Lett. 93, 143201 (2004).

[28] P. Naidon, S. Endo, and M. Ueda (2012), arXiv: 1208.3912,

[29] C. Chin (2011), arXiv:1111.1484.

[30] Y. Yamaguchi, Phys. Rev. 95, 1628 (1954).

[31] S. Moszkowski, S. Fleck, A. Krikeb, L. Theußl, J.-M. Richard, and K. Varga, Phys. Rev. A 62, 032504 (2000).

[32] M. D. Lee, T. Köhler, and P. S. Julienne, Phys. Rev. A 76, 012720 (2007).

[33] P. Naidon and M. Ueda, C. R. Phys. 12, 13 (2011).

[34] Smorodinsky, J. Phys. Acad. Sci. U.S.S.R. 8, 219 (1944).

[35] H. A. Bethe, Phys. Rev. 76, 38 (1949).

[36] P. Naidon, E. Hiyama, and M. Ueda, Phys. Rev. A 86, 012502 (2012).

[37] F. Ferlaino, A. Zenesini, M. Berninger, B. Huang, H.-C. Nägerl, and R. Grimm, Few-Body Syst. 51, 113 (2011).

[38] R. B. Wiringa, V. G. J. Stoks, and R. Schiavilla, Phys. Rev. C 51, 38 (1995).

[39] A. S. Jensen, E. Garrido, and D. V. Fedorov, Few-Body Syst. 22, 193 (1997).

[40] R. Schmidt, S. P. Rath, and W. Zwerger, Eur. Phys. J. B 85, 386 (2012).

[41] P. K. Sørensen, D. V. Fedorov, A. S. Jensen, and N. T. Zinner, Phys. Rev. A 86, 052516 (2012).

[42] The presence of the the factor $\frac{1}{2}$ is due to the conventional definition of the effective range.

[43] Such an approach can be applied, however, to the case of narrow Feshbach resonances (see Ref. [26]) for which the mechanism setting the three-body parameter is different than that of single-channel resonances considered here.

[44] A separable potential has only one two-body bound state. In our model, this state describes the two-body bound state of the original potential which is close to the twobody scattering threshold, while the other bound states of the potential are not included in our model. As a result, the trimer has no lower-lying state to dissociate to, and it appears as a true bound state, rather than a resonance.

[45] The same happens for potentials with finite support, although the effective range does not increase with the number of bound states in this case. 